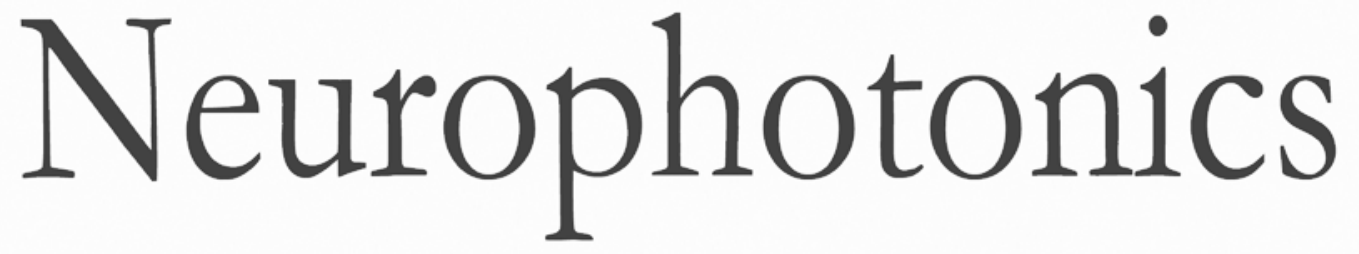

\title{
Study of memory deficit in Alzheimer's disease by means of complexity analysis of fNIRS signal
}

David Perpetuini

Roberta Bucco

Michele Zito

Arcangelo Merla 


\title{
Study of memory deficit in Alzheimer's disease by means of complexity analysis of fNIRS signal
}

\author{
David Perpetuini,, ${ }^{a, b, \star}$ Roberta Bucco, ${ }^{\mathrm{c}}$ Michele Zito, ${ }^{\mathrm{c}}$ and Arcangelo Merla ${ }^{\mathrm{a}, \mathrm{b}}$ \\ aUniversity G. d'Annunzio, Infrared Imaging Lab, Centro Institute for Advanced Biomedical Technologies, Chieti, Italy \\ bUniversity G. d'Annunzio, Department of Neurosciences, Imaging and Clinical Sciences, Chieti-Pescara, Italy \\ 'University G. d'Annunzio, Department of Medicine and Science of Ageing, Chieti-Pescara, Italy
}

\begin{abstract}
Working memory deficit is a signature of Alzheimer's disease (AD). The free and cued selective reminding test (FCSRT) is a clinical test that quantifies memory deficit for AD diagnosis. However, the diagnostic accuracy of FCSRT may be increased by accompanying it with neuroimaging. Since the test requires doctorpatient interaction, brain monitoring is challenging. Functional near-infrared spectroscopy (fNIRS) could be suited for such a purpose because of the fNIRS flexibility. We investigated whether the complexity, based on sample entropy and multiscale entropy metrics, of the fNIRS signal during FCSRT was correlated with memory deficit in early AD. fNIRS signals were recorded over the prefrontal cortex of healthy and early $A D$ participants. Group differences were tested through Wilcoxon-Mann-Whitney test $(p<0.05)$. At group level, we found significant differences for Brodmann areas 9 and 46. The results, although preliminary, demonstrate the feasibility of performing ecological studies on early AD with fNIRS. This approach may provide a potential neuroimaging-based method for diagnosis of early $A D$, viable at the doctor's office level, improving test-based diagnosis. The increased entropy of the fNIRS signal in early AD suggests the opportunity for further research on the neurophysiological status in AD and its relevance for clinical symptoms. $\odot 2017$ Society of Photo-Optical Instrumentation Engineers (SPIE) [DOI: 10.1117/1.NPh.5.1.011010]
\end{abstract}

Keywords: functional near-infrared spectroscopy; free and cued selective reminding test; Alzheimer's disease; entropy. Paper 17058SSRR received Apr. 28, 2017; accepted for publication Aug. 16, 2017; published online Sep. $26,2017$.

\section{Introduction}

Alzheimer's disease (AD) can be characterized by dementia that usually begins with a slight failure of memory that slowly becomes more acute. ${ }^{1}$ In fact, the International Working Group on AD's proposal for early diagnosis of AD considers the evidence of significant and progressive episodic memory deficit as the core diagnostic criterion. Thus, the aim of researchers is to find efficient tests to detect early memory impairment to be used in a clinical environment. Among the variety of tests to define memory impairment, the free and cued selective reminding test $(\text { FCSRT })^{2,3}$ is considered to be highly sensitive and specific in distinguishing $\mathrm{AD}$ patients from healthy controls ${ }^{4}$ and people affected by other forms of dementia. ${ }^{5}$ In addition, the test makes it possible to differentiate patients at an early stage of AD from mild cognitive impairment (MCI) patients' nonconverters. ${ }^{6}$ During this test, 12 pictures of everyday life are shown to the patient, which they are asked to memorize. After this encoding phase, the participant has to recall all the figures previously shown. If he is not able to remember all the figures by himself, a cued recall phase follows, during which the doctor reminds the participant the semantic field of each figure not retrieved. The same procedure is repeated after $30 \mathrm{~min}$. During this time, other tests are administered to the patient. Failures in recalling the pictures are considered highly significant for early memory impairment. However, despite the validity of the FCSRT, it could be very useful for the diagnosis of $\mathrm{AD}$ to overlap neuroimaging techniques with the administration of the test.

*Address all correspondence to: David Perpetuini, E-mail: david.perpetuini@ unich.it
So far, working memory (WM) has been investigated using different neuroimaging techniques, such as functional magnetic resonance imaging (fMRI) and positron emission tomography (PET) ${ }^{7,8}$ However, for the administration of the FCSRT, the doctor is required to sit next to the patient and interact with him. Therefore, it is not possible to perform the task in an fMRI scanner. Furthermore, performing this test lying down in a scanner would not be comfortable for the patient, and the result of the test could be invalidated. Moreover, this test is widely used for ambulatory patients, thus the employment of a portable technique is advantageous with respect to the fMRI.

Therefore, to preserve the interaction between the doctor and the patient, and the ecological conditions of the FCSRT, in this study we used functional near-infrared spectroscopy (fNIRS) as it is more suitable for this type of application.

fNIRS is a noninvasive hemodynamic-based neuroimaging technique that measures the relative changes in concentration of oxygenated $(\mathrm{HbO})$ and deoxygenated hemoglobin ( $\mathrm{Hbb}) \mathrm{sec}-$ ondary to neuronal activity. Given its better spatial resolution ${ }^{9}$ and lower sensitivity to motion artifacts compared to electroencephalography (EEG), and due to its portability and light weight, ${ }^{10}$ this technique is very suitable for ecological applications, such as a clinical interview.

fNIRS has already been proposed to study AD patients. For example, it was used to investigate visuospatial deficits ${ }^{11}$ and different brain responses to a verbal fluency task (VFT) in healthy individuals and AD patients. ${ }^{12-14}$ In particular, it was 
shown that a change in the cortex oxygenation could be due to an alteration of the regional brain functions or to deteriorated neurovascular coupling. In addition, fNIRS was also employed for monitoring the effect of cholinesterase inhibitors on $\mathrm{AD}$ patients during a VFT, ${ }^{15}$ demonstrating a variation in the oxygenation of the prefrontal cortex and the areas involved with speech processing. Furthermore, differences between healthy, MCI, and AD patients ${ }^{16}$ were investigated using fNIRS, making the potential relevance of this technique for the diagnosis of the AD undeniable.

Researchers argued that the complexity of a biological system output could change with age and disease. ${ }^{17-20}$ Complexity can be defined as the difficulty of predicting a signal. Along the same line, we hypothesized a change in fNIRS signal complexity due to neurovascular coupling dysregulation related to the task and to the disease. Thus, we investigated how the complexity of an fNIRS signal changed between healthy adults and patients. In particular, we used the sample entropy (SampEn $)^{21}$ and the multiscale entropy (MSE) ${ }^{22}$ as metrics for the complexity.

For instance, SampEn was used to study the nonlinear properties of heart rate (HR) time series ${ }^{23,24}$ in neonates to evaluate the reduced variability of HR in the course of neonatal sepsis, and in adults, to investigate the obstructive sleep apnea syndrome. ${ }^{25}$ In addition, it was used to evaluate the complexity of fMRI signal in patients affected by attention-deficit hyperactivity disorder ${ }^{26}$ and in patients with schizophrenia during a social task. ${ }^{27}$ With regards to $\mathrm{AD}$, it was used to analyze background brain activity through magnetoencephalography (MEG) ${ }^{18}$ and EEG signals. ${ }^{19,20}$

If we consider the fNIRS signal from an information viewpoint, it is a nonstationary and nonlinear time series and its entropy can thus be used to discriminate among different healthy brain states. In particular, in this study, a complexity analysis was carried out to distinguish early AD patients from healthy people. This problem has been tackled already by several researchers, but, to the best of our knowledge, this is the first time that this kind of analysis has been performed on fNIRS signals recorded during a WM test in completely ecological conditions. The possibility of using this complex analysis as an indicator of early $\mathrm{AD}$ could support the traditional clinical diagnosis of this dementia. Because of the dysregulation in neural responses in patients affected by $\mathrm{AD}$, we expected the patients with $\mathrm{AD}$ to exhibit higher complexity than healthy controls.

\section{Materials and Methods}

\subsection{Participants}

Eleven healthy participants (mean age \pm SD: $67.5 \pm 5.0$ years; $8 \mathrm{M} / 3 \mathrm{~F}$ ) and eleven early AD patients (mean age \pm SD: $72.2 \pm$ 4.5 years; $7 \mathrm{M} / 4 \mathrm{~F}$ ) voluntarily participated in this study. The inclusion criterion was a diagnosis of mild probable $\mathrm{AD}$, according to the Diagnostic and Statistical Manual of Mental Disorders, fifth edition. The exclusion criteria were: moderate-severe cognitive impairment (mini-mental state examination $<25 / 30$ ), ${ }^{28}$ vascular dementia (excluding those based on clinical and neuroimaging evaluation), behavioral disorders, hydrocephalus or space-occupying lesions documented by neuroimaging techniques, psychiatric disorders, or a history of stroke or traumatic brain injury. The Research Ethics Board of the University of Chieti-Pescara approved this study and it was performed in agreement with the Declaration of Helsinki. All participants signed informed consent and could withdraw from the study at any time.

\subsection{Free and Cued Selective Reminding Test}

This test was proposed by Buschke, ${ }^{3}$ and it was validated for AD diagnosis by Lemos et al. ${ }^{2}$ This test is composed of an encoding phase, in which the participant has to memorize different figures, and an immediate and a delayed recall during which the participant has to name the figures previously seen. In the encoding phase, 12 selected stimulus figures are presented, 4 at a time on a sheet, 1 figure per quadrant. The subject is required to indicate and mention aloud each illustration after the experimenter verbally discloses the semantic cue of each of them. After this phase, three immediate recall trials follow. Every trial is preceded by $30 \mathrm{~s}$ of countdown to prevent recall from short-term memory. In this phase, the subject is asked to freely recall the items within 2 min [immediate free recall (IFR)]; next, the experimenter reminds the subject of the semantic cues of any items not freely retrieved [immediate cued recall (ICR)]. If the participant does not remember the figures with the cue, the experimenter reminds the subject of the images, providing him with both the items and the cues. The total recall is the sum of the free and cued recall. After $30 \mathrm{~min}$, during which the subject has to perform nonverbal tasks, the same procedure of recall is carried out again [delayed free recall/delayed cued recall (DFR/ DCR)]. The nonverbal tests ${ }^{29}$ used to separate the immediate and the delayed recalls are: clock drawing test (CDT) ${ }^{30}$ digit span test (DST), ${ }^{31}$ Corsi span test (CST), ${ }^{32}$ trial making test (TMT) (versions A and B), ${ }^{33}$ and Benton test (BT). ${ }^{34}$

During the administration of FCSRT, a rest period (RP) of 1 min between two different tasks was added to the original structure of the test to reset the brain activity. The experimental design is shown in Fig. 1.

\subsection{Functional Near-Infrared Spectroscopy Measurement}

To measure the cerebral hemodynamic, a commercial frequencydomain near-infrared spectroscopy instrument (Imagent, ISS Inc., Champaign, Illinois) was used. This instrument is made up of 32 laser diodes sources, 16 emitting light at $690 \mathrm{~nm}$ and 16 at $830 \mathrm{~nm}$, and 4 photomultiplier tubes (PMT). The light is modulated at $110 \mathrm{MHz}$, while the PMTs are at $110.005 \mathrm{MHz}$. Time multiplexing was employed for the sources during the testing (switch mode: 16; sample frequency: $10 \mathrm{~Hz}$ ). The light power emitted by the sources on the scalp of the participants is lower than $4 \mathrm{~mW} / \mathrm{cm}^{2}$, with respect to the American National Standards Institute standard limits and allowed for safe acquisition.

Nine sources for each wavelength and four detectors were used, resulting in 17 measurement channels in a multidistance configuration ( 3 to $4 \mathrm{~cm}$ ). To ensure the comfort of the patients, a helmet with fixed holes for the fibers was used, positioned according to the international 10-20 electrodes placement system. ${ }^{35}$ The brain areas involved in memory deficits, as well as in impaired abstract thinking and inhibition, are Brodmann areas (BA) 8,9 , and $47 .{ }^{36}$ We tried to find the best compromise between covering the brain areas involved in this function and the fixed positions of the holes for the fibers. The best solution we found is presented in Fig. 2. The lowest detector is positioned in correspondence with the Fp point, according to the 10-20 system, ${ }^{35,38}$ and the channels cover BAs 8, 9, 10, and 46 . 


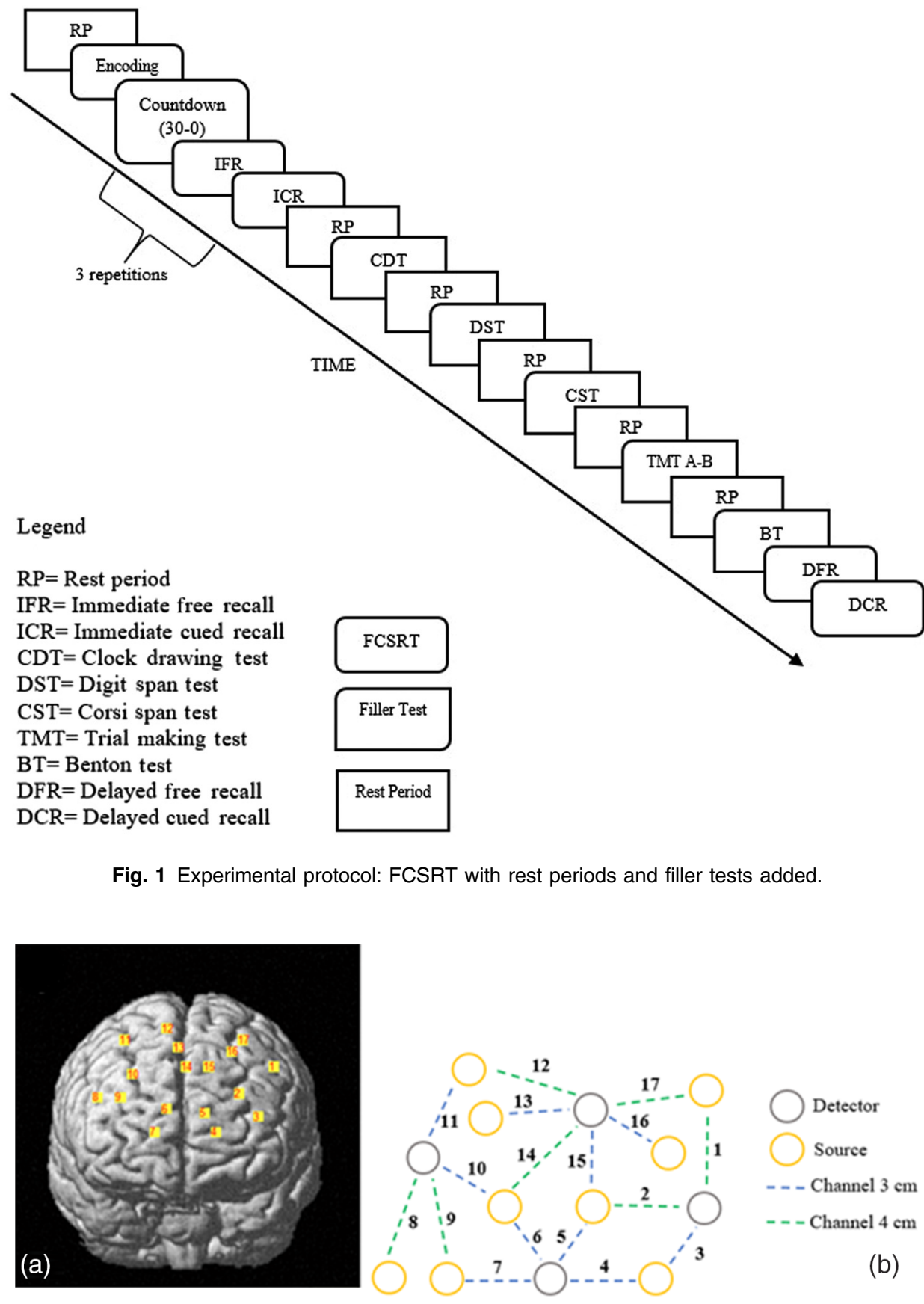

Fig. 2 (a) Channels configuration plot using NIRS-SPM software ${ }^{37}$ and (b) schematic optodes placement and channels distances.

\subsection{Processing of Functional Near-Infrared Spectroscopy Data}

The quality of the signals was first checked to discard channels with a low signal-to-noise ratio. The signals were checked by visual inspection and evaluating the correlation between $\mathrm{HbO}$ and $\mathrm{Hbb}$; if it was positive, the channel would have been discarded. All channels showed good scalp coupling due to the placement over the forehead, so none were discarded across all the participants. For the preprocessing, the Homer2 NIRS Processing package ${ }^{39}$ was used.

The intensity signals were converted into optical density (OD). Motion artifacts were identified as a signal change $>10 \%$ of the standard deviation (SD) over a period of $1 \mathrm{~s}$. These artifacts were corrected using a wavelet-based correction algorithm. ${ }^{40,41}$ The signals were bandpass filtered (third-order Butterworth filter, cutoff frequencies: 0.01 to $0.4 \mathrm{~Hz}$ ) to reduce systemic noises, such as heart and breathing rates. Then, the OD signals were converted into $\mathrm{HbO}$ and $\mathrm{Hbb}$ signals through the modified Beer-Lambert law (differential pathlengths factors $=6$ ).

\subsection{Calculation of Sample Entropy}

The complexity of a signal and the randomness of a process could be calculated by using several nonlinear time series analysis techniques, such as the computation of the entropy of the time series. Various typologies of this notion are known in literature. In this study, we used the SampEn, ${ }^{21}$ which is defined as 
the negative natural logarithm of the conditional probability that signals subseries of length $m$ (pattern length) that match pointwise within a tolerance $r$ (similarity factor) also match at the $m+1$ point. $^{22}$

The SampEn of a time series $\left\{x_{1}, \ldots x_{N}\right\}$ of length $N$ can be evaluated by the following set of equations: ${ }^{26}$

$\operatorname{SampEn}(m, r, N)=-\ln \left[\frac{U^{m+1}(r)}{U^{m}(r)}\right]$,

$U^{m}(r)=[N-m \tau]^{-1} \sum_{i=1}^{N-m \tau} C_{i}^{m}(r)$,

where

$C_{i}^{m}(r)=\frac{B_{i}}{N-(m+1) \tau}$,

where $B_{i}=$ number of $j$ where $d\left|X_{i}, X_{j}\right| \leq r, \quad X_{i}=$ $\left[x_{i}, x_{i+\tau} \ldots, x_{i+(m-1) \tau}\right], \quad X_{j}=\left[x_{j}, x_{j+\tau} \ldots, x_{j+(m-1) \tau}\right], \quad$ and $i \leq$ $j \leq N-m \tau, j \neq i$.

In the equations, $N$ is the number of the samples, $m$ is the length of the subseries considered, $r$ represents the tolerance within two subseries, which are considered equals, and $\tau$ is the time delay expressed in the samples.

It is possible to evaluate SampEn over different time scales using the MSE, after a coarse-graining procedure, hence using values of $\tau \neq 1$. This consists of creating a new time series composed of the mean value of a number of adjacent samples, without overlap, equal to the established scale factor. Then, it is possible to calculate the SampEn of each coarse-grained time series.

The procedure to create a course-grained series is described in the following equation: ${ }^{22}$

$y_{j}^{(\tau)}=\frac{1}{\tau} \sum_{i=(j-1) \tau+1}^{j \tau} x_{i} \quad 1 \leq j \leq \frac{N}{\tau}$.

This could discern stochastic and noisy deterministic time series, is quite robust for signals with a low level of noise and artifacts and gives meaningful information with a limited number of data samples. ${ }^{42}$

There are no guide lines agreed upon for choosing the best parameter to compute SampEn for a biomedical signal, but it is known from literature that a data length of $10^{m}$ to $20^{m}$ is necessary, and the similarity factor has to be chosen as a percentage of the SD of the signal under study (usually 0.1 to $0.35 \cdot \mathrm{SD}$ ). ${ }^{21}$ Thus, the correct choice of $\tau$ is important because the number of samples of the course-grained series has to be higher than $10^{m}$ to $20^{m}$, and the new sample frequency has to be higher than the frequencies of the harmonics of the signal. ${ }^{43}$

We evaluated the SampEn and MSE of each channel both for $\mathrm{HbO}$ and $\mathrm{Hbb}$ during the RP, IFR, and DFR phases. For the resting period and IFR, we evaluated the mean value of the SampEn and MSE computed for each repetition. According to literature, ${ }^{21}$ the parameters chosen for the evaluation of SampEn and MSE are

- $m=2$,

- $r=0.2 \mathrm{SD}$, and

- $\tau=[2,3]$.
Given the limited length of the IFR and DFR phases, it is impossible to investigate the complexity of the signal at larger scales. Our analysis was thus limited to shorter scales. The time durations of ICR and DCR are very brief, only a few seconds, and sometimes these phases are not present for healthy controls, making the evaluation of the SampEn and MSE of the fNIRS signal during these phases meaningless.

\subsection{Statistical Analysis}

Wilcoxon-Mann-Whitney tests were performed to compare the SampEn and MSE ( $\tau=2$ and 3 ) between the patients and healthy controls for the RP, IFR, and DFR $(p<0.05)$, for each channel's $\mathrm{HbO}$ and Hbb signals.

A false discovery rate (FDR) for multiple comparison correction $(q<0,05)$ was applied to correct the $p$-values of the independent Wilcoxon-Mann-Whitney tests in order to avoid false positives. This approach is widely used for neuroimaging data. It operates simultaneously on all channelwise test statistics. It defines a corrected threshold to control the error rate and to determine which tests have to be considered statistically significant. $^{44}$

The receiver operating characteristics (ROC) ${ }^{45}$ analysis was performed for SampEn and MSE values on channels showing significant differences between the control and AD groups. ROC analysis was performed using the International Business Machines Corporation Statistical Package for Social Sciences (SPSS 21.0; New York).

To homogenize the metric labels, in Sec. 3, we consider SampEn as MSE without resempling $(\tau=1)$.

\section{Results}

During the RP and IFR, the values of MSE $(\tau=1,2,3)$ do not show significant differences between healthy controls and patients for all of the channels. MSE $(\tau=1)$ and MSE $(\tau=2)$ during DFR show significant differences between the two groups for the channels reported in Table 1. After the FDR correction, only $\mathrm{HbO} \mathrm{Ch} 15$ and $\mathrm{Hbb} \mathrm{Ch} 8$ confirm statistical differences. Patients exhibit higher values of entropy than healthy controls, as shown in Fig. 3. MSE with scale $\tau=3$ does

Table 1 Area under the ROC curves for the channels that show significant difference between healthy controls and AD patients.

\begin{tabular}{|c|c|c|c|c|c|}
\hline \multirow[b]{2}{*}{ Hemoglobin } & \multirow[b]{2}{*}{ Entropy } & \multirow[b]{2}{*}{ Channels } & \multirow[b]{2}{*}{$A \cup C$} & \multicolumn{2}{|c|}{$\begin{array}{l}\text { Wilcoxon-Mann- } \\
\text { Whitney }(p<0.05)\end{array}$} \\
\hline & & & & $z$-scores & $\begin{array}{c}\text { Significance } \\
\text { (p-values) }\end{array}$ \\
\hline \multirow[t]{3}{*}{$\mathrm{HbO}$} & $\operatorname{MSE}(\tau=1)$ & Ch 8 & 0.785 & 2.2326 & 0.03 \\
\hline & $\operatorname{MSE}(\tau=2)$ & Ch 4 & 0.760 & 2.0356 & 0.04 \\
\hline & & $\operatorname{Ch} 15^{\mathrm{a}}$ & 0.826 & 2.5609 & 0.01 \\
\hline \multirow[t]{4}{*}{$\mathrm{Hbb}$} & $\operatorname{MSE}(\tau=1)$ & Ch 8 & 0.785 & 2.2326 & 0.03 \\
\hline & $\operatorname{MSE}(\tau=2)$ & Ch 4 & 0.769 & 2.1013 & 0.04 \\
\hline & & Ch $8^{a}$ & 0.818 & 2.4953 & 0.01 \\
\hline & & Ch 15 & 0.760 & 2.0356 & 0.04 \\
\hline
\end{tabular}



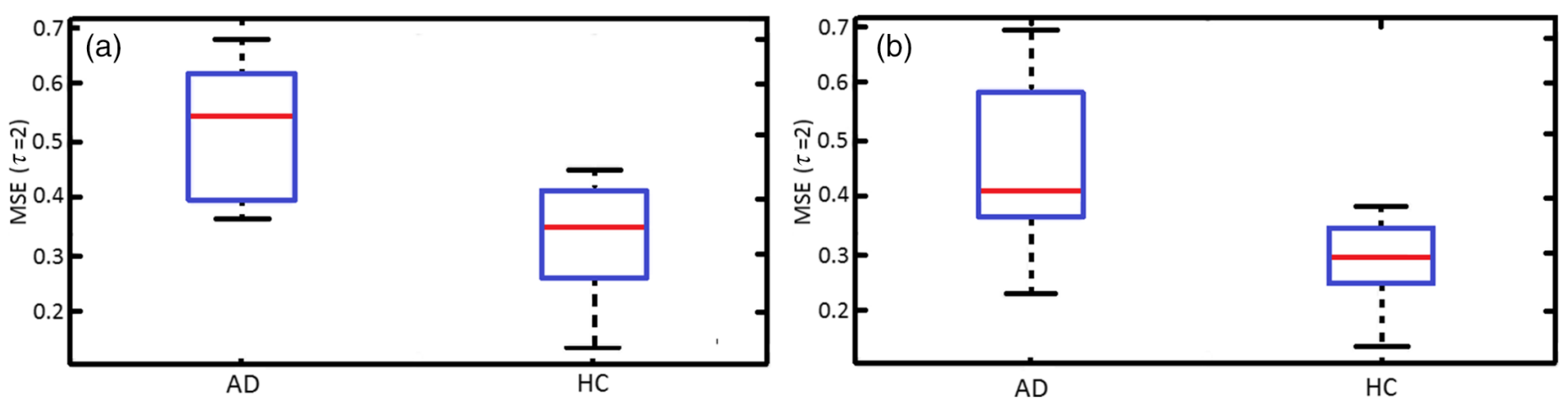

Fig. 3 Boxplot of MSE $(\tau=2)$ for (a) Ch 15 oxyhemoglobin during DFR and (b) Ch 8 deoxyhemoglobin during DFR.
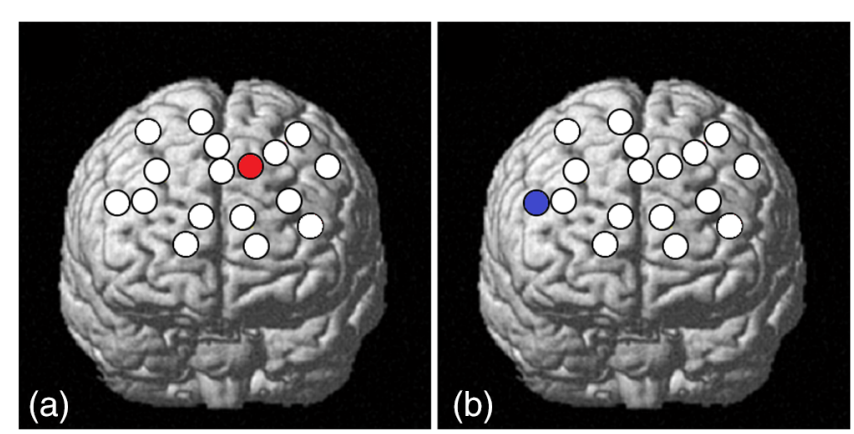

Fig. 4 Channels that show a significant difference between the two groups through Wilcoxon-Mann-Whitney test $(p<0.05)$ survived to FDR correction for (a) DFR, MSE $(\tau=2)$, oxyhemoglobin and (b) DFR, MSE $(\tau=2)$, deoxyhemoglobin.

not show significant differences between patients and healthy groups for DFR. Using Brodmann's Atlas, we found that channels that showed a significant difference between the two groups cover Broadmann areas 9 and 46. The areas of the brain for which there are distinctions between healthy controls and patients are shown in Fig. 4.

Table 1 reports the area under the ROC curve for the channels that exhibit significant differences between the two groups, and Fig. 5 shows the ROC curves underlying the higher area under the curve (AUC). They were obtained for Ch 10 during the DFR phase for $\operatorname{MSE}(\tau=2)$ on the Hbb signal (cutoff $=0.3843$, sensitivity $=0.727, \quad 1$-specificity $=0.091$ ) and for Ch 18 during the DFR phase for $\operatorname{MSE}(\tau=2)$ on $\mathrm{HbO}$ signal (cutoff $=0.3159, \quad$ sensitivity $=0.909$, 1 -specificity $=0.182$ ).

\section{Discussion}

The aim of this study is to demonstrate the feasibility of a complexity analysis approach for fNIRS signals collected during ecological conditions to discriminate healthy controls and $\mathrm{AD}$ patients. In particular, according to the hypothesis that aging and disease lead to different complexity outputs of a system, we analyzed the complexity of $\mathrm{HbO}$ and $\mathrm{Hbb}$ signals using SampEn and MSE algorithms during the IFR and DFR phases of the FCSRT for both healthy controls and patients. This was done to discriminate between the two groups.

Typically, block or repeated event paradigms are used for fNIRS studies and general linear model or block averagingbased analyses are carried out. Unfortunately, because of the ecological paradigm, this kind of analysis was not feasible in this study. In fact, the timing of the test is not fixed, but is subject dependent. In the FCSRT, only the maximum time of the free recalling phase is fixed, and its duration depends on the performance of the subjects. Moreover, the subject can name the pictures whenever he feels ready during the available time, without a predetermined timing. It is thus impossible to create a proper design matrix and properly model the expected functional response. Therefore, it is necessary to investigate the possibility
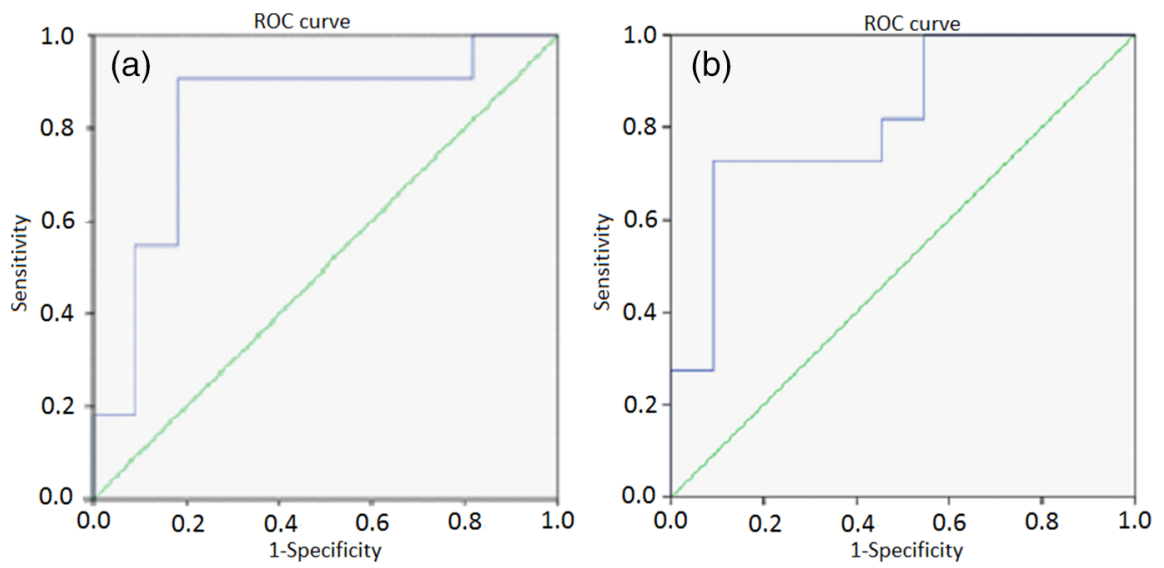

Fig. 5 (a) ROC curve for MSE ( $\tau=2$ ) of Ch 15 oxyhemoglobin during DFR and (b) ROC curve for MSE $(\tau=2)$ of Ch 8 deoxyhemoglobin during DFR. 
of using a different approach for signal analysis, such as a complexity investigation.

The main finding of the present study is that significant differences for $\operatorname{MSE}(\tau=1)$ and $\operatorname{MSE}(\tau=2)$ between $\operatorname{AD}$ patients and healthy controls performing the FCSRT, during the DFR phase, were observed in the salient region involved in this kind of task, as shown in Table 1. After the FDR correction, only $\operatorname{MSE}(\tau=2)$ gives some significant results. More precisely, patients with $\mathrm{AD}$ exhibit higher values of entropy than controls (Fig. 4). This analysis can discriminate BAs 9 and 46 (Fig. 4), regions involved in WM tasks. ${ }^{36}$ This result confirms the hypothesis that brain signals from AD constitute a dysregulation of neurological patterns and that there is an increase in complexity, as demonstrated by Vaillancourt and Newell. ${ }^{17}$ MSE with $\tau=3$ does not seem to be significant for this kind of analysis. This is probably caused by the limited number of samples or by the specific dysregulation of these brain areas, which have a temporal structure that requires shorter temporal scales.

Complexity analysis of brain signals for patients with AD has been already carried out by several authors. Gómez et al.$^{18}$ found that MEG signals from patients are less complex than controls during resting state. Deng et al. ${ }^{46}$ have the same findings on EEG signals. According to the literature, an increase in the complexity of fNIRS signal is observed during the performance of a mental arithmetic task ${ }^{47}$ and during a WM task ${ }^{48}$ with respect to the RP for healthy people. Conversely, we did not find significant differences between the two groups for SampEn and MSE during the rest phase. This might be due to the limited length of this phase and, therefore, the limited number of samples. On the contrary, the higher complexity of patients with respect to healthy people observed during DFR could be a result of a dysfunction in the neurovascular coupling in the frontal area due to the task and to the activation of these brain regions. In fact, cerebral blood flow (CBF) depends on the brain activity, and it is regulated by the coordinate action of neurons, glia, and vascular cells. When neurons are activated, various vascular changes are elicited and lead to CBF increases in the activated area. ${ }^{49}$ In AD patients, there is deposition of amyloid $\beta$-peptide in neuropil and vessels, so neurovascular coupling is disrupted and CBF is not appropriate to support the metabolic demand of the tissue. ${ }^{49}$

It is also possible that the increased complexity observed in our study is caused by a degradation of functional neural connectivity. For example, Pijnenburg et al. ${ }^{50}$ found a loss of connectivity during a WM task using MEG signals on AD patients. Stam et al. ${ }^{51}$ observed a loss of small-world network characteristics in AD patients using EEG, and Supekar et al. ${ }^{52}$ had the same findings in fMRI.

We found similar results for both $\mathrm{HbO}$ and $\mathrm{Hbb}$. This finding could be considered relevant because these two signals are physiologically anticorrelated, hence, their predictabilities should be almost the same. In fact, we would suppose to find similar values of entropy for both the time series.

The ROC analyses show that the MSE $(\tau=2)$ evaluated on Ch 8 and 15 can discriminate AD patients from healthy controls because of its higher area under ROC curve (Table 1). They are placed over BA 46 and BA 9, respectively.

Even though our results are preliminary, this study demonstrates that it is possible to use the SampEn and MSE to discriminate efficaciously healthy people from AD patients during ecological protocols. In particular, to the best of our knowledge, it is the first time that the fNIRS signal was collected during the administration of the FCSRT in a clinical condition, giving a physiological correlation to the diagnosis of $\mathrm{AD}$ obtained by this test. Due to fNIRS' portability and it being noninvasive, this technique could become an important support for the diagnosis of AD.

One limitation of our study is the limited sample size and the limited length of the fNIRS signal. However, it is due to the test's structure and to its ecological features. In fact, probably because of the restricted number of samples, we were not able to find differences during the RP. ${ }^{53}$ In our future studies, we will enlarge the sample size of the rest phase to obtain more useful information to better discriminate $\mathrm{AD}$ patients from healthy controls.

We will also combine fNIRS with other neuroimaging techniques to investigate which one would be more effective in assessing regional differences in the brain in terms of sensitivity and specificity. Furthermore, we plan to investigate the complexity of the $\mathrm{HbO}$ and $\mathrm{Hbb}$ signals by testing different parameters, in particular regarding the similarity factor, for the calculation of the entropy, in order to find the best parameters settings for $\mathrm{HbO}$ and $\mathrm{Hbb}$. In addition, it could be of great interest to study the systemic contribution to the complexity of the fNIRS signal, measuring some physiological signals (i.e., breathing rate and HR) and placing short separation channels.

\section{Conclusions}

To the best of our knowledge, this is the first study investigating the complexity of brain fNIRS signals in patients with AD during the administration of FCSRT, in completely ecological conditions.

The results show that MSE with scale $\tau=2$ is appropriate to investigate brain signal complexity for this kind of task. AD patients show higher values of entropy than healthy controls during DFR phase in BAs 9 and 46. These results are consistent with the hypothesis that $\mathrm{AD}$ may be characterized by a dysregulation of complex functional networks and support the hypothesis of a complexity variation with age or disease of biological systems.

\section{Disclosures}

There are no conflicts of interest, financial or otherwise, to declare by the authors.

\section{Acknowledgments}

We want to thank Paola Pinti and Damiano Formenti for proofreading the paper.

\section{References}

1. B. Dubois et al., "Research criteria for the diagnosis of Alzheimer's disease: revising the NINCDS-ADRDA criteria," Lancet Neurol. 6(8), 734-746 (2007).

2. R. Lemos et al., "The free and cued selective reminding test: validation for mild cognitive impairment and Alzheimer's disease," J. Neuropsychol. 9(2), 242-257 (2015).

3. H. Buschke, "Cued recall in amnesia," J. Clin. Neuropsychol. 6(4), 433-440 (1984).

4. E. Grober et al., "Free and cued selective reminding identifies very mild dementia in primary care," Alzheimer Dis. Assoc. Disord. 24(3), 284 (2010).

5. E. Grober et al., "Free and cued selective reminding distinguishes Alzheimer's disease from vascular dementia," J. Am. Geriatr. Soc. 56(5), 944-946 (2008). 
6. E. Grober and H. Buschke, "Genuine memory deficits in dementia," Dev. Neuropsychol. 3(1), 13-36 (1987).

7. M. Rajah and M. D'Esposito, "Region-specific changes in prefrontal function with age: a review of PET and fMRI studies on working and episodic memory," Brain 128(9), 1964-1983 (2005).

8. M. D'Esposito, B. R. Postle, and B. Rypma, "Prefrontal cortical contributions to working memory: evidence from event-related fMRI studies," Exp. Brain Res. 133(1), 3-11 (2000).

9. A. M. Chiarelli et al., "Combining energy and Laplacian regularization to accurately retrieve the depth of brain activity of diffuse optical tomographic data," J. Biomed. Opt. 21(3), 036008 (2016).

10. M. Ferrari and V. Quaresima, "A brief review on the history of human functional near-infrared spectroscopy (fNIRS) development and fields of application," NeuroImage 63(2), 921-935 (2012).

11. J. B. M. Zeller et al., "Altered parietal brain oxygenation in Alzheimer's diseaseas assessed with near-infrared spectroscopy," Am. J. Geriatr. Psychiatry 18(5), 433-441 (2010).

12. C. Hock et al., "Near infrared spectroscopy in the diagnosis of Alzheimer's diseaseas," Ann. N.Y. Acad. Sci. 777(1), 22-29 (1996).

13. A. J. Fallgatter et al., "Loss of functional hemispheric asymmetry in Alzheimer's dementia assessed with near-infrared spectroscopy," Cognit. Brain Res. 6(1), 67-72 (1997).

14. M. J. Herrmann et al., "Reduced prefrontal oxygenation in Alzheimer disease during verbal fluency tasks," Am. J. Geriatr. Psychiatry 16(2), 125-135 (2008)

15. F. G. Metzger et al., "Effects of cholinesterase inhibitor on brain activation in Alzheimer's patients measured with functional near-infrared spectroscopy," Psychopharmacology 232(23), 4383-4391 (2015).

16. H. Arai et al., "A quantitative near-infrared spectroscopy study: a decrease in cerebral hemoglobin oxygenation in Alzheimer's disease and mild cognitive impairment," Brain Cognit. 61(2), 189-194 (2006).

17. D. E. Vaillancourt and K. M. Newell, "Changing complexity in human behavior and physiology through aging and disease," Neurobiol. Aging 23(1), 1-11 (2002).

18. C. Gómez et al., "Analysis of MEG background activity in Alzheimer's disease using nonlinear methods and ANFIS," Ann. Biomed. Eng. 37(3), 586-594 (2009).

19. D. Abásolo et al., "Entropy analysis of the EEG background activity in Alzheimer's disease patients," Physiol. Meas. 27(3), 241-253 (2006).

20. J. Escudero et al., "Analysis of electroencephalograms in Alzheimer's disease patients with multiscale entropy," Physiol. Meas. 27(11), 10911106 (2006).

21. J. S. Richman and J. R. Moorman, "Physiological time-series analysis using approximate entropy and sample entropy," Am. J. Physiol.-Heart Circ. Physiol. 278(6), H2039-H2049 (2000).

22. M. Costa, A. L. Goldberger, and C.-K. Peng, "Multiscale entropy analysis of biological signals," Phys. Rev. E 71(2), 021906 (2005).

23. W.-S. Kim et al., "Nonlinear characteristics of heart rate time series: influence of three recumbent positions in patients with mild or severe coronary artery disease," Physiol. Meas. 26(4), 517-529 (2005).

24. D. E. Lake et al., "Sample entropy analysis of neonatal heart rate variability," Am. J. Physiol. Regul. Integr. Comp. Physiol. 283(3), R789R797 (2002).

25. H. M. Al-Angari and A. V. Sahakian, "Use of sample entropy approach to study heart rate variability in obstructive sleep apnea syndrome," IEEE Trans. Biomed. Eng. 54(10), 1900-1904 (2007).

26. M. O. Sokunbi et al., "Resting state fMRI entropy probes complexity of brain activity in adults with ADHD," Psychiatry Res.: Neuroimaging 214(3), 341-348 (2013).

27. M. O. Sokunbi et al., "Nonlinear complexity analysis of brain FMRI signals in schizophrenia," PLoS One 9(5), e95146 (2014).

28. M. F. Folstein, S. E. Folstein, and P. R. McHugh, "Mini-mental state: a practical method for grading the cognitive state of patients for the clinician," J. Psychiatr. Res. 12, 189-198 (1975).

29. H. Spinnler and G. Tognoni, "Standardizzazione e taratura Italiana di test neuropsicologici [Italian standardization of neuropsychological tests]," Italy J. Neurol. Sci. 8, 1-120 (1987).

30. T. Sunderland et al., "Clock drawing in Alzheimer's disease," J. Am. Geriatr. Soc. 37(8), 725-729 (1989).
31. A. Orsini et al., "Verbal and spatial immediate memory span: normative data from 1355 adults and 1112 children," Italy J. Neuro. Sci. 8(6), 537548 (1987).

32. R. P. C. Kessels et al., "The Corsi block-tapping task: standardization and normative data," Appl. Neuropsychol. 7(4), 252-258 (2000).

33. R. M. Reitan and D. Wolfson, The Halstead-Reitan Neuropsycholgical Test Battery: Therapy and Clinical Interpretation, Neuropsychological Press, Tucson, Arizona (1985).

34. L. Arthur, "A visual retention test for clinical use," Arch. Neurol. Psychiatry 54(3), 212-216 (1945).

35. R. W. Homan, J. Herman, and P. Purdy, "Cerebral location of international 10-20 system electrode placement," Electroencephalogr. Clin. Neurophysiol. 66(4), 376-382 (1987).

36. M. Catani et al., "Beyond cortical localization in clinico-anatomical correlation," Cortex 48(10), 1262-1287 (2012).

37. J. C. Ye et al., "NIRS-SPM: statistical parametric mapping for nearinfrared spectroscopy," NeuroImage 44(2), 428-447 (2009).

38. A. M. Chiarelli et al., "Comparison of procedures for co-registering scalp-recording locations to anatomical magnetic resonance images," J. Biomed. Opt. 20(1), 016009 (2015).

39. T. J. Huppert et al., "HomER: a review of time-series analysis methods for near-infrared spectroscopy of the brain," Appl. Opt. 48(10), D280D298 (2009).

40. B. Molavi and G. A. Dumont, "Wavelet-based motion artifact removal for functional near-infrared spectroscopy," Physiol. Meas. 33(2), 259270 (2012).

41. A. M. Chiarelli et al., "A kurtosis-based wavelet algorithm for motion artifact correction of fNIRS data," NeuroImage 112, 128-137 (2015).

42. X.-S. Zhang and R. J. Roy, "Derived fuzzy knowledge model for estimating the depth of anesthesia," IEEE Trans. Biomed. Eng. 48(3), 312 323 (2001).

43. M. Aboy et al. "Characterization of sample entropy in the context of biomedical signal analysis," in 29th Annual Int. Conf. of the IEEE Engineering in Medicine and Biology Society (EMBS '07), IEEE (2007).

44. C. R. Genovese, N. A. Lazar, and T. Nichols, "Thresholding of statistical maps in functional neuroimaging using the false discovery rate," NeuroImage 15(4), 870-878 (2002).

45. M. H. Zweig and G. Campbell, "Receiver-operating characteristic (ROC) plots: a fundamental evaluation tool in clinical medicine," Clin. Chem. 39(4), 561-577 (1993).

46. B. Deng et al., "Complexity extraction of electroencephalograms in Alzheimer's disease with weighted-permutation entropy," Chaos 25(4), 043105 (2015).

47. K. Çiftçi et al. "Complexity analysis of functional near-infrared spectroscopy signals." in 13th European Signal Processing Conf., IEEE (2005).

48. T. Angsuwatanakul, K. Iramina, and B. Kaewkamnerdpong, "Brain complexity analysis of functional near infrared spectroscopy for working memory study," in 8th Biomedical Engineering International Conference (BMEiCON), IEEE (2015).

49. H. Girouard and C. Iadecola, "Neurovascular coupling in the normal brain and in hypertension, stroke, and Alzheimer disease," J. Appl. Physiol. 100(1), 328-335 (2006).

50. Y. A. L. Pijnenburg et al., "EEG synchronization likelihood in mild cognitive impairment and Alzheimer's disease during a working memory task," Clin. Neurophysiol. 115(6), 1332-1339 (2004).

51. C. J. Stam et al., "Small-world networks and functional connectivity in Alzheimer's disease," Cereb. Cortex 17(1), 92-99 (2006).

52. K. Supekar et al., "Network analysis of intrinsic functional brain connectivity in Alzheimer's disease," PLoS Comput. Biol. 4(6), e1000100 (2008).

53. M. O. Sokunbi, "Sample entropy reveals high discriminative power between young and elderly adults in short fMRI data sets," Front. Neuroinf. 8, 69 (2014).

Biographies for the authors are not available. 